\title{
INEQUALITIES FOR A PERTURBATION THEOREM OF PALEY AND WIENER
}

ROBERT M. YOUNG

\begin{abstract}
A classical theorem of Paley and Wiener states that the set of functions $\left\{e^{i \lambda_{n} t}\right\}_{n=-\infty}^{\infty}$ forms a basis for $L^{2}(-\pi, \pi)$ whenever the following condition is satisfied:
\end{abstract}

$$
\left\|\sum c_{n}\left(e^{i \lambda_{n} t}-e^{i n t}\right)\right\|^{2} \leqq \theta^{2} \sum\left|c_{n}\right|^{2} \quad(0 \leqq \theta<1) .
$$

It is known that (*) holds whenever $\lambda_{n}$ is real and $\left|\lambda_{n}-n\right| \leqq L<1$ $(-\infty<n<\infty)$, and may fail to hold if $\left|\lambda_{n}-n\right|=\frac{1}{4}$.

In this note we show, more generally, that the condition $\left|\lambda_{n}-n\right|<1$ is also insufficient to ensure (*).

1. Introduction. One of the fundamental results from the theory of nonharmonic Fourier series states that the functions $e^{i \lambda_{n} t}(-\infty<$ $n<\infty)$ form a basis for $L^{2}(-\pi, \pi)$ if they satisfy an inequality of the form

$$
\left\|\sum c_{n}\left(e^{i \lambda_{n} t}-e^{i n t}\right)\right\|^{2} \leqq \theta^{2} \sum\left|c_{n}\right|^{2}
$$

for some $\theta(0 \leqq \theta<1)$ and all finite sequences $\left\{c_{n}\right\}$ [7, p. 109]. It is well known $[8$, p. 210] that condition (1) holds whenever

$$
\left|\lambda_{n}-n\right| \leqq L<(\log 2) / \pi \quad(-\infty<n<\infty) .
$$

In another direction, Levinson showed [6, p. 48] that if

$$
\left|\lambda_{n}-n\right| \leqq L<\frac{1}{4},
$$

then every function in $L^{2}(-\pi, \pi)$ has a nonharmonic series expansion $f \sim \sum c_{n} e^{i \lambda_{n} t}$ which is equiconvergent with its ordinary Fourier series over any interval $[-\pi+\varepsilon, \pi-\varepsilon]$ for any positive $\varepsilon$. For $\lambda_{n}$ real, the question of whether (2) implies (1) was answered in the affirmative by M. I. Kadec [5].

The purpose of this note is to show that the condition $\left|\lambda_{n}-n\right|<\frac{1}{4}$ is not sufficient to imply (1). Our proof is based on the result [6, p. 67]

Received by the editors May 3, 1973 and, in revised form, July 25, 1973.

AMS (MOS) subject classifications (1970). Primary 42A60; Secondary 42A64.

Key words and phrases. Nonharmonic Fourier series, frames, functions of exponential type.

(c) American Mathematical Society 1974 
that if $\left\{\mu_{n}\right\}$ is given by

$$
\begin{aligned}
\mu_{n} & =n-\frac{1}{4}, & & n>0, \\
& =0, & & n=0, \\
& =n+\frac{1}{4}, & & n<0,
\end{aligned}
$$

then $\left\{e^{i \mu_{n} t}\right\}_{n \neq 0}$ is closed in $L^{2}(-\pi, \pi)$, and therefore (1) cannot hold with $\lambda_{n}=\mu_{n}$. Using the same example, Ingham had already established in $[4$, p. 378] that the inequality

$$
A \sum\left|c_{n}\right|^{2} \leqq\left\|\sum c_{n} e^{i \mu_{n} t}\right\|^{2}
$$

cannot hold for any positive $A$, thereby also showing that (1) fails for this choice of $\lambda_{n}$. In this paper, the following theorem is established.

THEOREM. If $\left\{\mu_{n}\right\}$ is given by (3) and if $\left\{\lambda_{n}\right\}$ is a sequence of complex numbers for which $\left|\lambda_{n}-\mu_{n}\right| \rightarrow 0(n \rightarrow \pm \infty)$, then (1) cannot hold.

COROLLARY. The condition $\left|\lambda_{n}-n\right|<\frac{1}{4}$ is not sufficient to ensure (1).

2. Fourier frames. Duffin and Schaeffer [3, p. 343] have termed a set of functions $\left\{e^{i \lambda_{n} t}\right\}$ a frame over the interval $(-\gamma, \gamma)$ if there exist positive constants $A$ and $B$ such that

$$
A \int_{-\gamma}^{\gamma}|g(t)|^{2} d t \leqq \frac{1}{2 \pi} \sum_{n}\left|\int_{-\gamma}^{\gamma} g(t) e^{i \lambda_{n} t} d t\right|^{2} \leqq B \int_{-\gamma}^{\gamma}|g(t)|^{2} d t
$$

for every function $g(t)$ in $L^{2}(-\gamma, \gamma)$. It follows from a theorem of Paley and Wiener [7, p. 13] that an equivalent characterization is that the inequalities

$$
A \int_{-\infty}^{\infty}|f(x)|^{2} d x \leqq \sum_{n}\left|f\left(\lambda_{n}\right)\right|^{2} \leqq B \int_{-\infty}^{\infty}|f(x)|^{2} d x
$$

hold for every function $f$ which is entire of exponential type $\gamma$ and such that $f(x) \in L^{2}(-\infty, \infty)$. It is clear from (4) that a frame is a complete set of functions in $L^{2}(-\gamma, \gamma)$.

The following lemmas were established in [3, pp. 346, 360].

LEMMA 1. The removal of a vector from a frame leaves either a frame or an incomplete set.

Lemma 2. If $\left\{e^{i \lambda_{n} t}\right\}$ is a frame over $(-\gamma, \gamma)$, then there exists a $\delta>0$ such that $\left\{e^{i \gamma_{n} t}\right\}$ is a frame over the same interval whenever $\left|\gamma_{n}-\lambda_{n}\right| \leqq \delta$.

3. Proof of the Theorem. Let us suppose to the contrary that (1) does hold for some $\theta, 0 \leqq \theta<1$. Then $\left\{e^{i \lambda_{n} t}\right\}$ is a frame over the interval $(-\pi, \pi)$ [1]. By Lemma 2 , there is a $\delta>0$ such that $\left\{e^{i \gamma_{n} t}\right\}$ is a frame over $(-\pi, \pi)$ whenever $\left|\gamma_{n}-\lambda_{n}\right| \leqq \delta$. Since $\left|\lambda_{n}-\mu_{n}\right| \rightarrow 0$, it follows that, for 
some sufficiently large $N$, the set

$$
\left\{e^{i \lambda_{n} t}\right\}_{|n| \leqq N} \cup\left\{e^{i \mu_{n} t}\right\}_{|n|>N}
$$

is a frame over the same interval.

It is well known [2, p. 98] that if $f$ is entire of exponential type $\gamma$ and if $f(x) \in L^{2}(-\infty, \infty)$, then

It follows from (5) that the set

$$
|f(x+i y)|^{2} \leqq e^{2 \gamma|y|} \int_{-\infty}^{\infty}|f(x)|^{2} d x .
$$

$$
\left\{e^{i \lambda_{n} t}\right\}_{n=-N}^{N} \cup\left\{e^{i \mu_{n} t}\right\}_{n=-\infty}^{\infty}
$$

is also a frame over $(-\pi, \pi)$. Since $\left\{e^{i \mu_{n} t}\right\}_{n \neq 0}$ is closed in $L^{2}(-\pi, \pi)$, repeated application of Lemma 1 shows that the set $\left\{e^{i \mu_{n} t}\right\}_{n \neq 0}$ must be a frame over $(-\pi, \pi)$. Again invoking Lemma 2 , we get $\varepsilon>0$ such that the set $\left\{e^{ \pm i \gamma_{n} t}\right\}_{n=1}^{\infty}$ is a frame over $(-\pi, \pi)$ whenever $\left|\gamma_{n}-\mu_{n}\right| \leqq \varepsilon$. We complete the proof by showing that this leads to a contradiction.

Let us form the function $F(z)=\prod_{n=1}^{\infty}\left(1-z^{2} / \gamma_{n}^{2}\right)$. Then $F$ is entire of exponential type $\pi$ [2, p. 186], and it was shown by Levinson [6, p. 49] that if $\left\{\gamma_{n}\right\}$ satisfies the inequality $\left|\gamma_{n}-n\right| \leqq L<\frac{1}{4}$, then $F(x) \in L^{2}(-\infty, \infty)$. Therefore, under these conditions, $\left\{e^{i \gamma_{n} t}\right\}_{n \neq 0}$ is not closed in $L^{2}(-\pi, \pi)$ and therefore cannot possibly be a frame.

\section{REFERENCES}

1. R. P. Boas, Jr., A trigonometric moment problem, J. London Math. Soc. 14 (1939), 242-244. MR 1, 52.

2. - Entire functions, Academic Press, New York, 1954. MR 16, 914.

3. R. J. Duffin and A. C. Schaeffer, A class of nonharmonic Fourier series, Trans. Amer. Math. Soc. 72 (1952), 341-366. MR 13, 839.

4. A. E. Ingham, Some trigonometrical inequalities with applications to the theory of series, Math. Z. 41 (1936), 367-379.

5. M. I. Kadec, The exact value of the Paley-Wiener constant, Dokl. Akad. Nauk SSSR 155 (1964), 1253-1254=Soviet Math. Dokl. 5 (1964), 559-561. MR 28 \#5289.

6. N. Levinson, Gap and density theorems, Amer. Math. Soc. Colloq. Publ., vol. 26, Amer. Math. Soc., Providence, R.I., 1940. MR 2, 180.

7. R. Paley and N. Wiener, Fourier transforms in the complex domain, Amer. Math. Soc. Colloq. Publ., vol. 19, Amer. Math. Soc., Providence, R.I., 1934.

8. F. Riesz and B. Sz.-Nagy, Leçons d'analyse fonctionnelle, 2nd ed., Akad. Kiadó, Budapest, 1953; English transl., Functional analysis, Ungar, New York, 1955. MR 15, $132 ; 17,175$.

Department of Mathematics, Oberlin College, Oberlin, Ohio 44074 